\title{
OXIGENOTERAPIA DOMICILIAR: \\ PERFIL DOS USUÁRIOS ASSISTIDOS PELO \\ PROGRAMA MELHOR EM CASA
}

\author{
HOME OXYGEN THERAPY: \\ PROFILE OF USERS ASSISTED BY THE \\ MELHOR EM CASA PROGRAM
}

\section{OXIGENOTERAPIA EN EL HOGAR: PERFIL DE USUARIOS ASISTIDOS POR EL PROGRAMA MELHOR EM CASA}

\author{
Lívia Krever de Souza ${ }^{1}$ \\ Alisia Helena Weis ${ }^{2}$ \\ Carine Raquel Blatt ${ }^{3}$
}

Como citar este artigo: Souza LK, Weis AH, Blatt CR. Oxigenoterapia domiciliar: perfil dos usuários assistidos pelo Programa Melhor em Casa. Rev baiana enferm. 2021;35:e45064.

Objetivo: descrever o perfil dos usuários de oxigenoterapia domiciliar assistidos pelo Programa Melhor em Casa. Método: estudo transversal com coleta de dados retrospectiva e análise descritiva de prontuários dos usuários de oxigenoterapia domiciliar de um município do interior do Rio Grande do Sul. Resultados: dentre os 59 usuários cadastrados no programa para uso da oxigenoterapia domiciliar, identificaram-se portadores de Doença Pulmonar Obstrutiva Crônica $(n=35)$, cuidados paliativos $(n=13)$ e outras patologias $(n=11)$, idade média de 69,3 anos, sexo feminino (52,5\%), oxigenoterapia intermitente (57,6\%), tabagismo pregresso (71,2\%), tabagismo atual ( $8,5 \%)$, tempo médio de uso da oxigenoterapia de 17 meses. Como desfecho, foi identificado: morte (45,7\%), alta (11,9\%) e em uso de oxigenoterapia (40,7\%). Conclusão: os usuários de oxigenoterapia domiciliar assistidos pelo Programa Melhor em Casa do município estudado apresentavam idade avançada, sexo feminino, ex-tabagistas, baixa escolaridade, doença pulmonar obstrutiva crônica como doença de base e cuidados prestados por cuidadores familiares.

Descritores: Oxigenoterapia. Serviços de Assistência Domiciliar. Cuidado de Enfermagem. Atenção Primária à Saúde. Doença Pulmonar Obstrutiva Crônica.

Objective: to describe the profile of home oxygen therapy users assisted by the Melhor em Casa Program. Method: cross-sectional study with retrospective data collection and descriptive analysis of medical records of home oxygen therapy users in a municipality in the countryside of Rio Grande do Sul. Results: among the 59 users enrolled in the program for the use of home oxygen therapy, Patients with Chronic Obstructive Pulmonary Disease ( $n=35)$, palliative care $(n=13)$ and other pathologies $(n=11)$, mean age of 69.3 years, female gender $(52.5 \%)$, intermittent oxygen therapy (57.6\%), previous smoking (71.2\%), current smoking (8.5\%), mean time of oxygen therapy use of 17 months were identified. As an outcome, death (45.7\%), discharge (11.9\%) and oxygen therapy (40.7\%) were identified.

\footnotetext{
Enfermeira. Universidade Federal de Ciências da Saúde de Porto Alegre. Porto Alegre, Rio Grande do Sul, Brasil. liviakrever@hotmail.com. https://orcid.org/00000003-4512-0922.

2 Enfermeira. Doutora em Enfermagem. Docente da Universidade Federal das Ciências da Saúde de Porto Alegre. Porto Alegre, Rio Grande do Sul, Brasil. https://orcid.org/0000-0003-4830-4583.

3 Farmacêutica. Doutora em Farmácia. Docente da Universidade Federal das Ciências da Saúde de Porto Alegre. Porto Alegre, Rio Grande do Sul, Brasil. https://orcid.org/0000-000 I-5935-1196.
} 
Conclusion: home oxygen therapy users assisted by the Melhor em Casa Program of the municipality studied had advanced age, female, ex-smokers, low schooling, chronic obstructive pulmonary disease as an underlying disease and care provided by family caregivers.

Descriptors: Oxygen Therapy. Home Care Services. Nursing Care. Primary Health Care. Chronic Obstructive Pulmonary Disease.

Objetivo: describir el perfil de los usuarios de oxigenoterapia domiciliaria asistidos por el Programa Melhor em Casa. Método: estudio transversal con recolección retrospectiva de datos y análisis descriptivo de registros médicos de usuarios domiciliarios de oxigenoterapia en un municipio del interior de Rio Grande do Sul. Resultados: entre los 59 usuarios inscritos en el programa para el uso de oxigenoterapia domiciliaria, se identificaron pacientes con Enfermedad Pulmonar Obstructiva Crónica $(n=35)$, cuidados paliativos $(n=13)$ y otras patologias $(n=11)$, edad media de 69,3 años, sexo femenino (52,5\%), oxigenoterapia intermitente (57,6\%), tabaquismo previo (71,2\%), tabaquismo actual (8,5\%), tiempo medio de uso de oxigenoterapia de 17 meses. Como resultado, se identificaron la muerte (45,7\%), el alta (11,9\%) y la oxigenoterapia (40,7\%). Conclusión: los usuarios de oxigenoterapia domiciliaria atendidos por el Programa Melhor em Casa del municipio estudiado fueron mujeres de edad avanzada, exfumadores, baja escolaridad, enfermedad pulmonar obstructiva crónica como enfermedad subyacente y atención brindada por cuidadores familiares.

Descriptores: Oxigenoterapia. Servicios de Atención Domiciliaria. Cuidados de Enfermería. Atención Primaria de Salud. Enfermedad Pulmonar Obstructiva Crónica.

\section{Introdução}

A oxigenoterapia domiciliar, caracterizada como método de suporte ventilatório, é geralmente prescrita aos pacientes que apresentam a cronificação da hipoxemia e disponibilizada aos pacientes em Atenção Domiciliar (AD), que necessitam de oferta de oxigênio em maiores concentrações do que a disponível no ar ambiente $^{(1-2)}$. A AD é uma estratégia adotada pelo Ministério da Saúde (MS) para fornecer maior acesso aos cuidados em saúde aos usuários portadores de doenças crônicas. Tem como objetivos reduzir a taxa de ocupação dos leitos hospitalares, promover um cuidado adaptado às necessidades dos usuários e, ainda, garantir a autonomia do paciente ${ }^{(3)}$.

A oferta da oxigenoterapia domiciliar tornou-se mais efetiva com a implantação da $\mathrm{AD}$, possibilitando a correção nas alterações clínicas decorrentes da insuficiência de oxigênio no sangue e, consequentemente, a melhora da qualidade de vida dos usuários com doenças respiratórias crônicas ${ }^{(1)}$. A oxigenoterapia domiciliar é prescrita, na maioria das vezes, aos pacientes portadores de Doença Pulmonar Obstrutiva Crônica (DPOC) em estágio avançado, grau III e IV na escala da Global Initiative For Chronic Obstructive Lung Disease ${ }^{(2,4)}$. Além disso, a oxigenoterapia possibilita o acompanhamento no domicílio de pacientes em cuidados paliativos que necessitam dessa terapia ${ }^{(1,5)}$.

A falta de orientações para o uso correto da oxigenoterapia domiciliar pode resultar em consequências graves e danosas à saúde dos usuários $^{(6)}$. Os principais riscos da oxigenoterapia podem ser classificados como riscos biológicos, físicos e funcionais ${ }^{(2-3)}$. Os riscos biológicos são ocasionados por incêndios e explosões; os riscos físicos, por traumas ocasionados pelo cateter ou máscaras e ressecamento de secreções devido à umidificação inadequada ${ }^{(3)}$. Por fim, os riscos funcionais, causam diversas complicações, como retenção de $\mathrm{CO}_{2}$, atelectasias, aumento da pressão arterial sistêmica, redução do débito cardíaco, aumento da pressão arterial e riscos de toxicidade, apresentados por manifestações citotóxicas do oxigênio ${ }^{(3,6)}$.

O Programa Melhor em Casa, criado pelo MS em 2011, é um programa de AD que contempla ações e medidas de prevenção, tratamento de doenças, reabilitação, paliação e promoção da saúde $^{(3)}$. Na cidade em que foi realizado este estudo, foi implantado em 2011 e a oxigenoterapia domiciliar é fornecida pela Secretaria Municipal de Saúde. A Equipe Multidisciplinar de Atenção 
Domiciliar (EMAD) é responsável pela instalação, reposição e assistência aos usuários em uso da oxigenoterapia domiciliar, recebendo suporte das Estratégias Saúde da Família do município.

Não obstante a oxigenoterapia domiciliar fazer parte dos recursos terapêuticos ofertados pelo Sistema Único de Saúde (SUS) há mais de 10 anos, pouco se conhece sobre o perfil dos pacientes que o utilizam. O conhecimento do perfil dos usuários e suas necessidades é importante para o planejamento do cuidado e para o desenvolvimento de atividades de educação em saúde dos usuários e cuidadores no que diz respeito à utilização da oxigenoterapia domiciliar. Dessa forma, o objetivo deste estudo é descrever o perfil dos usuários de oxigenoterapia domiciliar assistidos pelo Programa Melhor em Casa.

\section{Método}

Trata-se de estudo transversal com coleta de dados retrospectiva e análise de dados descritiva $^{(7-8)}$. Utilizou-se dados primários extraídos dos prontuários dos usuários de oxigenoterapia domiciliar assistidos pelo Programa Melhor em Casa de um município localizado na Região Metropolitana de Porto Alegre (RS).

A coleta de dados ocorreu entre setembro e outubro de 2020, na sede da EMAD do município, no qual a EMAD é composta por médico, enfermeiro, técnicos de enfermagem, fisioterapeuta e condutores/instaladores de oxigênio. Estes profissionais prestam assistência aos pacientes com moderada complexidade de cuidados, no horário das $7 \mathrm{~h}$ às $13 \mathrm{~h}$ e das $15 \mathrm{~h}$ às $21 \mathrm{~h}$, todos os dias da semana. O município possui uma população em torno de 40 mil habitantes, conforme estimativa de 2019 do Instituto Brasileiro de Geografia e Estatística (IBGE) ${ }^{(9)}$.

A população estudada foi composta por todos os usuários de oxigenoterapia domiciliar assistidos pelo Programa Melhor em Casa do município, no período de dezembro de 2018 a janeiro de 2020. A escolha desse período está relacionada ao início dos registros nos prontuários pela EMAD. Antes de dezembro de 2018, a maioria dos usuários não possuía prontuário.
Após janeiro de 2020, ocorreu a troca de profissionais da EMAD, e estes tiveram dificuldades para dar continuidade aos registros. Foram excluídos da pesquisa os usuários cujos prontuários possuíam dados incompletos.

As variáveis investigadas estavam baseadas nos indicadores estabelecidos pelo $\mathrm{MS}^{(10)}$ para o Programa Melhor em Casa, tais como: sexo, idade, escolaridade, cor, situação conjugal, patologia de base para indicação do uso de oxigênio, presença de outras comorbidades, procedência do encaminhamento, tabagismo, etilismo, mobilidade, cuidadores, forma de uso da oxigenoterapia, média de horas/dia de uso de oxigênio intermitente, tempo médio de internação, tipo de fonte de oxigênio e desfecho.

Os dados foram coletados em instrumento construído no Microsoft Word® e tabulados em uma planilha do Microsoft Excel ${ }^{\circledR}$. As variáveis foram apresentadas por meio de estatística descritiva simples, em número absoluto e percentual.

O estudo foi aprovado pelo Comitê de Ética em Pesquisa (CEP) da Universidade Federal de Ciências da Saúde de Porto Alegre (UFCSPA) com o Certificado de Apresentação de Apreciação Ética (CAAE) n. 35421720.6.0000.5345.

\section{Resultados}

No período investigado, 64 usuários foram cadastrados para uso de oxigenoterapia domiciliar. Destes, cinco foram excluídos da pesquisa pela ausência das variáveis investigadas nos registros dos prontuários. Portanto, os resultados do estudo referem-se a 59 usuários.

Na Tabela 1 são apresentadas as características sociodemográficas dos usuários. Identificou-se uma média de idade superior a 60 anos, com uma amplitude de 1 a 93 anos. Os dois usuários com idade inferior a 30 anos faziam uso da oxigenoterapia por cardiopatia congênita grave e paralisia infantil. Houve um discreto predomínio do sexo feminino, cor branca, escolaridade de ensino fundamental, estado marital casado, familiar como cuidador, usuários restritos ao leito ou acamados. Entre os cuidadores que eram familiares, o sexo feminino predominou. 
Tabela 1 - Características sociodemográficas dos usuários de oxigenoterapia domiciliar. Município da Região Metropolitana de Porto Alegre, Rio Grande do Sul, Brasil - dezembro 2018 a março 2020. $(\mathrm{N}=59)$

\begin{tabular}{|c|c|c|}
\hline Variáveis & $\mathbf{n}$ & $\%$ \\
\hline \multicolumn{3}{|l|}{ Média de idade (+Desvio Padrão) } \\
\hline Todos os usuários $(\mathrm{n}=59)$ & $69,3 \pm 18,3$ & \\
\hline Portadores de Doença Pulmonar Obstrutiva Crônica $(n=35)$ & $76,4 \pm 9,1$ & \\
\hline Cuidados paliativos $(\mathrm{n}=13)$ & $65,1 \pm 13,3$ & \\
\hline Outras patologias $(1)(n=11)$ & $56,9 \pm 25,3$ & \\
\hline \multicolumn{3}{|l|}{ Sexo } \\
\hline Feminino & 31,0 & 52,5 \\
\hline Masculino & 28,0 & 47,4 \\
\hline \multicolumn{3}{|l|}{ Cor } \\
\hline Branca & 54,0 & 91,5 \\
\hline Preta & 3,0 & 5,1 \\
\hline Parda & 2,0 & 3,4 \\
\hline \multicolumn{3}{|l|}{ Escolaridade } \\
\hline Não se aplica(2) & 2,0 & 4,4 \\
\hline Não frequentou a escola - analfabeto(a) & 4,0 & 6,8 \\
\hline Ensino fundamental completo/incompleto & 39,0 & 66,1 \\
\hline Ensino médio completo/incompleto & 12,0 & 20,3 \\
\hline Graduação completa/incompleta & 2,0 & 3,4 \\
\hline \multicolumn{3}{|l|}{ Situação conjugal } \\
\hline Não se aplica(2) & 2,0 & 3,4 \\
\hline Solteiro(a) & 4,0 & 6,8 \\
\hline Casado(a) & 34,0 & 57,6 \\
\hline Divorciado(a) & 4,0 & 6,8 \\
\hline Viúvo(a) & 15,0 & 25,4 \\
\hline \multicolumn{3}{|l|}{ Cuidadores } \\
\hline Não possuem (autocuidado) & 16,0 & 27,1 \\
\hline Cuidadores contratados & 3,0 & 5,1 \\
\hline Cuidador de Instituição de Longa Permanência & 1,0 & 1,7 \\
\hline Técnico de enfermagem & 1,0 & 1,7 \\
\hline Familiares & 38,0 & 64,4 \\
\hline Filhas & 17,0 & 42,3 \\
\hline Esposas & 12,0 & 20,3 \\
\hline
\end{tabular}

Fonte: Elaboração própria.

(1) Outras patologias: sequelas de tuberculose $(n=3)$, insuficiência cardíaca congestiva $(n=2)$, broncopneumonia de repetição $(n=2)$, hipertensão pulmonar $(n=1)$, paralisia cerebral $(n=1)$, cardiopatia congênita $(n=1)$ e vasculopatia periférica $(n=1)$.

(2) Não se aplica: criança 11 meses de idade, cardiopatia congênita $(n=1)$, adolescente 13 anos com paralisia cerebral ( $n=1)$.

Conforme pode ser observado na Tabela 2, a DPOC foi a principal doença de base para a hipoxemia, seguida de cuidado paliativo: câncer de pulmão, câncer de cérebro, câncer de cabeça e pescoço e câncer de mama. Outras doenças crônicas foram identificadas no grupo investigado, tais como hipertensão arterial sistêmica, diabetes mellitus, cardiopatias e depressão. O tempo médio de uso da oxigenoterapia domiciliar por usuários portadores de DPOC foi de 23 meses. Dentre estes, 16 permaneciam em uso. Para os usuários em cuidados paliativos, o tempo médio foi de 5 meses, variando de 2 a 10 meses. O encaminhamento para uso da oxigenoterapia domiciliar ocorreu predominantemente do ambiente hospitalar. Identificou-se como desfechos: morte, alta e em uso de oxigenoterapia. 
Tabela 2 - Características clínicas dos usuários de oxigenoterapia domiciliar. Município da Região Metropolitana de Porto Alegre, Rio Grande do Sul, Brasil - dezembro 2018 a março 2020. ( $N=59)$

(continua)

\begin{tabular}{|c|c|c|}
\hline Variáveis & $\mathbf{n}$ & $\%$ \\
\hline \multicolumn{3}{|c|}{ Patologia de base para indicação do uso de oxigenoterapia } \\
\hline Doença Pulmonar Obstrutiva Crônica & 35,0 & 59,3 \\
\hline Cuidados paliativos & 13,0 & 22,0 \\
\hline Outras patologias(1) & 11,0 & 18,6 \\
\hline \multicolumn{3}{|l|}{ Presença de outras comorbidades } \\
\hline $\operatorname{Sim}(2)$ & 43,0 & 72,9 \\
\hline Não & 16,0 & 27,1 \\
\hline \multicolumn{3}{|l|}{ Procedência do encaminhamento } \\
\hline Ambulatório/Pneumologista & 12,0 & 20,3 \\
\hline Hospital do município & 22,0 & 37,3 \\
\hline Hospitais de outras cidades & 20,0 & 33,9 \\
\hline Posto de saúde & 5,0 & 8,5 \\
\hline \multicolumn{3}{|l|}{ Tabagismo } \\
\hline Tabagista atual & 5,0 & 8,5 \\
\hline Tabagista pregresso & 42,0 & 71,2 \\
\hline Não tabagista & 12,0 & 20,3 \\
\hline \multicolumn{3}{|l|}{ Etilismo } \\
\hline Etilista atual & 2,0 & 3,4 \\
\hline Etilista pregresso & 12,0 & 20,3 \\
\hline Não etilista & 45,0 & 76,3 \\
\hline \multicolumn{3}{|l|}{ Mobilidade } \\
\hline Deambulam & 26,0 & 44,1 \\
\hline Deambulam com auxílio & 18,0 & 30,5 \\
\hline Restrito ao leito & 3,0 & 5,1 \\
\hline Acamado & 11,0 & 18,6 \\
\hline Não se aplica(3) & 1,0 & 1,7 \\
\hline \multicolumn{3}{|l|}{ Uso da oxigenoterapia } \\
\hline Uso contínuo (24h/dia) & 25,0 & 42,4 \\
\hline Uso intermitente & 34,0 & 57,6 \\
\hline \multicolumn{3}{|c|}{$\begin{array}{l}\text { Média de horas/dia de uso de oxigenoterapia intermitente } \\
\text { (+Desvio Padrão) }\end{array}$} \\
\hline Todos os usuários $(n=34)$ & $9,3 \pm 5,4$ & \\
\hline Doença Obstrutiva Crônica $(n=25)$ & $10,2 \pm 5,8$ & \\
\hline Cuidado paliativo $(n=3)$ & $8,6 \pm 4,1$ & \\
\hline Outras patologias(1) $(n=6)$ & $6,8 \pm 4,3$ & \\
\hline \multicolumn{3}{|c|}{$\begin{array}{l}\text { Tempo médio de uso da oxigenoterapia domiciliar (meses) } \\
\text { (+Desvio Padrão) }\end{array}$} \\
\hline Todos os usuários $(n=59)$ & $17,0 \pm 17,4$ & \\
\hline Doença Obstrutiva Crônica $(n=35)$ & $23,0 \pm 19,5$ & \\
\hline Cuidado paliativo $(n=13)$ & $5,0 \pm 4,2$ & \\
\hline Outras patologias $(n=11)$ & $11,0 \pm 5,9$ & \\
\hline \multicolumn{3}{|l|}{ Tipo de fonte de Oxigênio } \\
\hline Cilindro & 40,0 & 67,8 \\
\hline Cilindro + válvula aspiração & 4,0 & 6,8 \\
\hline Cilindro + concentrador & 12,0 & 20,3 \\
\hline Concentrador & 3,0 & 5,1 \\
\hline \multicolumn{3}{|l|}{ Desfecho } \\
\hline Em uso de oxigenoterapia & 24,0 & 40,7 \\
\hline Alta & 7,0 & 11,9 \\
\hline
\end{tabular}


Tabela 2 - Características clínicas dos usuários de oxigenoterapia domiciliar. Município da Região Metropolitana de Porto Alegre, Rio Grande do Sul, Brasil - dezembro 2018 a março 2020. ( $N=59)$

(conclusão)

\begin{tabular}{lcc}
\hline Variáveis & $\mathbf{n}$ & $\mathbf{\%}$ \\
\hline Desfecho & 1,0 & 1,7 \\
$\quad$ Mudança de município & 27,0 & 45,7 \\
$\quad$ Óbito & & 28,8 \\
Usuários que permanecem em uso de oxigenoterapia & 17,0 & - \\
$\quad$ Doença Obstrutiva Crônica & - & 13,5 \\
Cuidado paliativo & 8,0 & 22,0 \\
Outras patologias(1) & & 20,0 \\
Usuários que foram a óbito & 13,0 & 1,7 \\
$\quad$ Doença Obstrutiva Crônica & 13,0 & 1,0 \\
Cuidado paliativo & & \\
Insuficiência cardíaca congestiva & & \\
\hline
\end{tabular}

Fonte: Elaboração própria.

Notas: Sinal convencional utilizado:

- Dado numérico igual a zero não resultante de arredondamento.

(1) Outras patologias: sequelas de tuberculose $(n=3)$, insuficiência cardíaca congestiva ( $n=2)$, broncopneumonia de repetição $(n=2)$, hipertensão pulmonar $(n=1)$, paralisia cerebral $(n=1)$, cardiopatia congênita $(n=1)$ e vasculopatia periférica $(n=1)$.

(2) Hipertensão arterial sistêmica, diabetes Mellitus, cardiopatias e depressão foram as principais doenças reportadas pelos pacientes.

(3) Não se aplica: criança 11 meses de idade, cardiopatia congênita $(n=1)$, adolescente 13 anos com paralisia cerebral ( $n=1$ ).

\section{Discussão}

Este estudo mostrou o perfil dos usuários em oxigenoterapia domiciliar de um município da região metropolitana do Rio Grande do Sul, gerando informações sociodemográficas e clínicas. Pôde-se identificar que a utilização da oxigenoterapia é mais frequente em usuários portadores de doenças respiratórias crônicas, principalmente a DPOC, pacientes em cuidados paliativos e aqueles com sequelas da tuberculose.

As doenças crônicas são caracterizadas pela progressão lenta e de longa duração. São doenças que ocorrem com o tempo. As características dos pacientes deste estudo, bem como demonstrado em outros estudos ${ }^{(11-12)}$, apontaram que a maioria dos usuários da oxigenoterapia possuía 60 anos ou mais, branco e do sexo feminino.

No município estudado, estima-se cerca de 158,8 usuários de oxigenoterapia para 100 mil habitantes. Essa prevalência pode ser muito variável e pode estar associada à prevalência das doenças de base, como a DPOC e o câncer. Estudo realizado em 16 países da Europa, nos anos de 2001 e 2002, indicou a prevalência de 6,6 usuários para 100 mil habitantes em uso de oxigenoterapia domiciliar, chegando a 240 para 100 mil habitantes nos Estados Unidos ${ }^{(13)}$.

A exposição à fumaça de tabaco, poluições do ar, fatores genéticos, envelhecimento, sexo feminino, bronquite, história de infecções frequentes e status socioeconômico são fatores de risco para DPOC ${ }^{(2,14)}$. Tais fatores podem aumentar a frequência de pacientes com DPOC nos municípios brasileiros e, consequentemente, o uso da oxigenoterapia domiciliar.

A análise da mobilidade dos usuários de oxigenoterapia mostrou que, para grande parte deles, estava prejudicada, limitando-os ao leito ou à cama. Diante desse dado, percebe-se que grande parte dos usuários de oxigenoterapia domiciliar necessita de cuidadores. Neste estudo, ficou evidenciado que as mulheres são cuidadoras em maior número. Esse predomínio é também apontado em outros estudos ${ }^{(13,15)}$. Esse fato ainda ocorre possivelmente pela construção social que, historicamente, atribui à mulher o papel de cuidadora ${ }^{(16)}$.

A pobreza e a baixa escolaridade fazem parte dos fatores de risco para DPOC, não estando definido, no entanto, se reflete a exposição aos poluentes, nutrição inadequada, infecções ou 
outros fatores relacionados ao perfil socioeconômico ${ }^{(2,17)}$. Em estudo de coorte realizado nos Estados Unidos ${ }^{(18)}$, a baixa renda foi relacionada com maior gravidade da doença e obstáculo no diagnóstico e tratamento adequado. Neste estudo, a maioria dos usuários apresentou baixo nível de escolaridade, corroborando os achados do estudo realizado pela Universidade Federal de São Paulo no ambulatório de oxigenoterapia domiciliar, em que 63,7\% dos usuários possuíam escolaridade de nível fundamental ${ }^{(18)}$.

Nos usuários desta pesquisa, verificou-se a presença de muitas doenças crônicas concomitantes à DPOC, tais como hipertensão arterial sistêmica, diabetes Mellitus, cardiopatias e depressão. A presença de outras doenças crônicas associadas à DPOC contribui para o aumento da morbidade e da mortalidade desses usuários ${ }^{(2)}$.

Neste estudo, para os usuários de oxigenoterapia intermitente, portadores de DPOC, a média foi de 10,2 horas por dia, percebendo-se que alguns usuários não utilizaram a terapia de maneira eficaz. Para usuários com DPOC, a utilização por 15 horas/dia ou mais de oxigenoterapia diminui a mortalidade e melhora a qualidade de vida ${ }^{(2,14)}$.

De acordo com a orientação do MS, a oxigenoterapia domiciliar está indicada aos pacientes com hipoxemia que preencham os critérios a seguir: não fazer uso do tabaco, PAO2 inferior a $55 \mathrm{mmHg}$ ou $\mathrm{SpO} 2$ inferior a $88 \%$, ou $\mathrm{PaO} 2$ entre 55 e $59 \mathrm{mmHg}$ ou SpO2 inferior a $89 \%$ e presença de hipertensão arterial pulmonar ${ }^{(2,14)}$. Neste estudo, o tabagismo pregresso está presente em grande parte dos usuários com DPOC, entretanto, em alguns usuários de oxigenoterapia, o tabagismo permanece. Esse dado também foi observado no estudo desenvolvido no município de Ribeirão Preto (SP), em que $30 \%$ dos usuários eram tabagistas ${ }^{(12)}$.

O tabagismo, além ser um dos principais fatores de risco para DPOC, afeta significativamente a qualidade de vida dos indivíduos portadores da doença. A cessação do tabaco é um dos critérios para a prescrição da oxigenoterapia domiciliar, pois seu uso reduz, de maneira relevante, o benefício da terapia ${ }^{(2,6)}$. A equipe da EMAD orienta usuários e cuidadores sobre a importância da cessação do tabagismo para a melhora do quadro do paciente, efetividade da oxigenoterapia, bem como esclarece que é pré-requisito para o recebimento desse método terapêutico. Apesar disso, nem todos os pacientes seguem essas recomendações.

A dispneia é reportada por $65 \%$ dos doentes em cuidados paliativos nas semanas que precedem a morte. No caso dos pacientes em fase terminal, a oxigenoterapia domiciliar possibilita que sejam assistidos no domicílio ${ }^{(19)}$. Esta terapia, no cuidado paliativo, não possui tanta eficácia como a utilização de opioides, no controle da dispneia; por esse motivo, deve ser utilizada somente após a tentativa de outras opções terapêuticas, farmacológicas e não farmacológicas ${ }^{(20)}$. Além disso, a oxigenoterapia é indicada em fase final de vida, nos seguintes casos: em doenças cardíacas, ao apresentar saturação <88\% na prova da marcha; angina não tratável com terapêutica médica otimizada; hipertensão pulmonar grave; insuficiência cardíaca congestiva grave; nos portadores de neoplasia terminal, se existir a evidência de hipoxemia e esperança de vida menor que seis meses ${ }^{(19,21)}$.

A oxigenoterapia domiciliar pode ser fornecida por meio de concentradores que captam o ar ambiente através de peneiras moleculares e o filtram, removendo o nitrogênio e aumentando a concentração de oxigênio. Estes equipamentos funcionam ligados à rede elétrica ${ }^{(22)}$. Também podem ser fornecidos por cilindros que necessitam ser recarregados e não dependem de energia elétrica. Com relação aos custos, estudo aponta que o fornecimento de oxigênio por concentradores pode propiciar redução de 54\% nos custos em relação aos cilindros, sendo que, nesse cálculo, não foram incluídos os gastos com energia elétrica ${ }^{(23)}$. Importante esclarecer que existem iniciativas que reduzem o valor do tratamento, a exemplo da Portaria Interministerial MME/GM n. 630, de 2011, que instituiu a tarifa social de energia elétrica para pessoas de baixa renda ${ }^{(23-24)}$.

A inadequada administração de oxigênio aos usuários portadores de doenças causadoras de hipoxemia pode agravá-las no decorrer do 
tempo. Dentre os sintomas, destacam-se: dificuldade respiratória, parestesia das extremidades do corpo, inquietação, fadiga, tosse seca, mal-estar e tontura ${ }^{(18)}$. A superoxigenação está entre as possíveis complicações relacionadas ao uso inadequado da terapia, nos casos de DPOC exacerbado, uma vez que, na tentativa de auxiliar o paciente, familiares podem ofertar-lhe volumes altos de $\mathrm{O}_{2}$, agravando a acidose respiratória e a hipercapnia ${ }^{(15,24)}$. Esta última ocorre pelo aumento, de forma descompensada, da pressão parcial de dióxido de carbono $\left(\mathrm{pCO}_{2}\right)$. Isso pode fazer com que o indivíduo venha a óbito ou apresente riscos de atelectasias ${ }^{(2,15,25)}$. Soma-se a isso, os riscos de toxicidade de traumas e explosões - em caso de usuários fumantes -, além de ressecamento de secreção provocado pela umidificação inadequada do $\mathrm{O}_{2}$ e, ainda, o desperdício, se o volume desse elemento for usado indevidamente pelo usuário ${ }^{(24)}$.

Desse modo, orientações sobre o uso dos medicamentos, volumes e tempo de oxigênio prescritos pelo médico e conscientização para a cessação do tabaco são essenciais para a efetividade da terapia. A higienização e a troca da água dos umidificadores deve ser diária, pois o volume da água deve estar dentro dos níveis demonstrados no produto. Os cateteres e extensores devem ser substituídos de acordo com o tipo utilizado, a fim de evitar a obstrução ${ }^{(6,19)}$.

Os cilindros devem ser fixos, com utilização de carrinhos ou correntes, sendo necessário atentar para a medida informada no manômetro, para evitar o término em horários sem possibilidade de reposição ${ }^{(22)}$. Quanto aos concentradores, os cuidados compreendem, principalmente: a colocação distante $15 \mathrm{~cm}$ da parede, para que consiga capturar o ar ambiente; atenção à indicação de troca de filtro; higiene do equipamento; e ser conectado à fonte de energia adequada ${ }^{(22)}$.

Além disso, deve-se verificar frequentemente a oximetria de pulso do paciente, realizar consultas rotineiras com médico e enfermeira, para atualização do quadro clínico, bem como para avaliação da necessidade de mudanças da terapia de oxigênio domiciliar ${ }^{(15,18,24)}$. As orientações sobre os cuidados necessários para o uso correto da oxigenoterapia domiciliar são fundamentais para pacientes e cuidadores efetivarem a terapia ${ }^{(19,25)}$.

Como limitação do estudo cita-se o fato de ter sido realizado com base nos prontuários dos pacientes em oxigenoterapia domiciliar e algumas informações podiam estar incompletas. Além disso, a amostra consistiu em um grupo de pacientes de um município no Sul do Brasil. Por isso, os dados podem não refletir a realidade de outros municípios brasileiros.

Diante do exposto, espera-se que os resultados deste estudo, associados com outros estudos epidemiológicos, possibilitem compreender e reconhecer as características dos cuidadores e usuários de oxigenoterapia domiciliar no município estudado, a fim de colaborar para ações de educação em saúde e prevenção de riscos relacionados à terapia.

\section{Conclusão}

Conclui-se que, entre as principais características dos usuários de oxigenoterapia domiciliar assistidos pelo Programa Melhor em Casa do município estudado, predominaram a idade avançada, sexo feminino, ex-tabagistas, baixa escolaridade, DPOC como doença de base e cuidados prestados por cuidadores familiares. $\mathrm{O}$ fornecimento da oxigenoterapia era feito predominantemente por meio de cilindros.

O serviço de saúde deve conhecer o perfil dos usuários, para que possa adaptar-se à realidade vivenciada por eles. Dessa forma, as informações apresentadas neste estudo são importantes, para que a equipe multiprofissional implemente novas ações ou intensifique outras já realizadas, como as ações educativas para prevenção de agravos relacionados aos riscos e/ou ao manejo inadequado da terapia, garantindo a efetividade do tratamento. Para os pacientes com DPOC, educação em saúde para a cessação do tabagismo e orientação de tempo de uso da oxigenoterapia ainda são um desafio. Para os usuários em cuidados paliativos, o conforto do paciente e a família devem ser prioridades na orientação. 
Por fim, sugere-se uma análise relacionada aos custos, para o município, com o fornecimento da terapia, principalmente relacionados aos cilindros, que possuem um custo significativamente superior, quando comparado aos concentradores.

\section{Colaborações:}

1 - concepção, projeto, análise, e interpretação dos dados: Lívia Krever de Souza, Alisia Helena Weis e Carine Raquel Blatt;

2 - redação do artigo, revisão crítica relevante do conteúdo intelectual: Livia Krever de Souza, Alisia Helena Weis e Carine Raquel Blatt;

3 - aprovação final da versão a ser publicada: Lívia Krever de Souza, Alisia Helena Weis e Carine Raquel Blatt.

\section{Referências}

1. Adde FV, Alvarez AE, Barbisan BN, Guimarães BR. Recomendações para oxigenoterapia domiciliar prolongada em crianças e adolescentes. J Pediatr. 2013;89(1):6-17. DOI: https://doi.org/10.1016/j.jped. 2013.02.003

2. Global Initiative for Chronic Obstructive Lung Disease. Global Strategy for the Diagnosis, Management, and Prevention of Chronic Obstructive Pulmonary Disease - 2019 Report [Internet]. Bethesda (EUA); 2019 [cited 2019 Jan 10]. Available from: https://goldcopd.org/ wp-content/uploads/2018/11/GOLD-2019-v1.7FINAL-14Nov2018-WMS.pdf

3. Brasil. Ministério da Saúde. Portaria no 825 , de 25 de abril de 2016. Redefine a Atenção Domiciliar no âmbito do Sistema Único de Saúde (SUS) e atualiza as equipes habilitadas [Internet]. Diário Oficial da União. Brasília (DF); 2016 [cited 2020 Aug 17]. Available from: https:// bvsms.saude.gov.br/bvs/saudelegis/gm/2016/ prt0825_25_04_2016.html

4. Fernandes FLA, Cukier A, Camelier AA, Fritscher CC, Costa CH, Pereira EDB, et al. Recomendações para o tratamento farmacológico da DPOC: perguntas e respostas. J bras pneumol. 2017;43(4):290-301. DOI: https://doi.org/10.1590/ s1806-37562017000000153

5. Vasconcelos GB, Pereira PM. Cuidados paliativos em atenção domiciliar: uma revisão bibliográfica.
Rev Adm Saúde. 2018;18(70). DOI: http://dx.doi. org/10.23973/ras.70.85

6. The Emergency Care Research Institute - ECRI. Ask HRC: Preventing Medical Air and Oxygen Delivery Errors [Internet]. Plymouth Meeting, PA: ECRI Institute; 2018 Feb 5 [cited 2020 Aug 15]. Available from: https://www.ecri.org/ search-results/member-preview/hrc/pages/ askhrc020518

7. Goldim JR. Manual de iniciação à pesquisa em saúde. Porto Alegre (RS): Dacasa; 2000.

8. Prodanov CF, Freitas EC. Metodologia do trabalho científico: métodos e técnicas da pesquisa e do trabalho acadêmico. $2 \mathrm{a}$ ed. Novo Hamburgo (RS): Feevale; 2013

9. Instituto Brasileiro de Geografia e Estatística. Panorama: Charqueadas [Internet]. Rio de Janeiro; 2010 [cited 2020 Aug 11]. Available from: https://cidades.ibge.gov.br/brasil/rs/charqueadas/ panorama

10. Brasil. Ministério da Saúde. Portaria GM n ${ }^{\circ}$ 1.600, de 7 de julho de 2011. Reformula a Política Nacional de Atenção às Urgências e institui a Rede de Atenção às Urgências no Sistema Único de Saúde. Brasília (DF); 2011 [cited 2020 Jul 25]. Available from: http://bvsms.saude.gov.br/bvs/ saudelegis/gm/2011/prt1600_07_07_2011.html

11. Kovelis D, Cruz PL, Silva LI, Serra JR, Sandoval PRM, Valderramas S. Características de usuários de oxigenoterapia domiciliar de longa duração no município de Curitiba, Brasil. Fisioter mov. 2019;32(5):e003204. DOI: http://dx.doi.org/10.1590/1980-5918.032.ao04

12. Bhatt SP, Balte PP, Schwartz JE, Cassano PA, Couper D, Jacobs Jr DR, et al. Discriminative Accuracy of FEV1: FVC Thresholds for COPD-Related Hospitalization and Mortality. JAMA. 2019;321(24):2438-47. DOI: http://doi:10. 1001/jama.2019.7233

13. Watanabe CS, Andrade LFC, Silva Neto MQ, Santos SFT, Kawatay LS. Oxigenoterapia domiciliar prolongada: perfil dos usuários e custos. Rev Enferm UERJ. 2015;23(1):95-101. DOI: https://doi. org/10.12957/reuerj.2015.7117

14. Lloyd-Owen SJ, Donaldson GC, Ambrosino N, Escarabill J, Farre R, Fauroux B, et al. Patterns of home mechanical ventilation use in Europe: results from the Eurovent survey. Eur Respir J. 2005;25(6):1025-31. DOI: http://doi:10.1183/0903 1936.05.00066704 
15. Oliveira NA, Souza EN, Brigola AG, Rossetti ES, Terassi M, Luchesi BM, et al. Idosos cuidadores em diferentes arranjos de moradia: comparação do perfil de saúde e de cuidado. Rev Gaúcha Enferm. 2019; 40:e20180225. DOI: https://doi. org/10.1590/1983-1447.2019.20180225

16. Peña A, Machado A, Mur T, Saiz M. Las mujeres siguen siendo las principales cuidadoras de pacientes dependientes. Aten Prim. 2018;50(1): 66-7. DOI: https://doi.org/10.1016/j.aprim.2017. 02.014

17. Brasil. Ministério da Saúde. Departamento de Atenção Básica. Caderno de Atenção Domiciliar [Internet]. Brasília (DF); 2013 [cited 2020 Aug 11]. Available from: https://bvsms.saude.gov.br/bvs/ publicacoes/caderno_atencao_domiciliar_melhor_ casa.pdf

18. Eisner MD, Blanc PD, Omachi TA, Yelin EH, Sidney S, Katz PP, et al. Socioeconomic status, race and COPD health outcomes. J Epidemiol Community Health. 2011;65(1):26-34. DOI: http:// dx.doi.org/10.1136/jech.2009.089722

19. Cedano S, Belasco AGS, Traldi F, Machado CLO, Bettencourt ARC. Influência das características sociodemográficas e clínicas e do nível de dependência na qualidade de vida de pacientes com DPOC em oxigenoterapia domiciliar prolongada. J bras pneumol. 2012;38(3):331-8. DOI: http://dx.doi.org/10.1590/S1806-3713201200 0300008

20. Frade L, Carreira N, Tosatto V, Marote S, Galriça Neto I. Oxygen Therapy in Advanced Disease: Do You Know the Evidence? Medicina Interna. 2019;26(4):51-6. DOI: http://dx.doi.org/ 10.24950/rspmi/Revisao/75/19/4/2019
21. Campbell ML, Yarandi H, Dove-Medows E. Oxygen is nonbeneficial for most patients who are near death. J Pain Symptom Manage. 2013 Oct;45:517-23. DOI: http://doi:10.1016/j. jpainsymman.2012.02.012

22. Gippsland Region Palliative Care Consortium, Clinical Practice Group. Oxygen Use in Palliative Care Guideline and Flowchart [Internet]. Australia; 2016 [cited 2020 Aug 15]. Available from: http://www.grpcc.com.au/wp-content/uploads/ 2014/04/GRPCC-CPG0041.02011-Oxygen-UseGuideline-and-Flowchart.pdf

23. Donoso MTV, Silqueira SMF, Barbosa RCGA, Vasconcelos TRC, Anastácio VLA. Oxigenoterapia e ventilação mecânica em atenção domiciliar [Internet]. Belo Horizonte (MG): Nescon UFMG; 2013 [cited 2020 Aug 16]. Available from: https://www.nescon.medicina.ufmg.br/biblioteca/ imagem/4259.pdf

24. Brasil. Ministério da Saúde. Secretaria de Atenção à Saúde. Departamento de Atenção Básica. Manual Instrutivo do Melhor em Casa [Internet]. Brasília (DF); 2012 [cited 2020 Aug 15]. Available from: http://189.28.128.100/dab/docs/ geral/cartilha_melhor_em_casa.pdf

25. Roncally SRO, Costa R, Granito TR, Vieira M, Nunes P, Luige M, et al. DPOC: oxigenioterapia e seus benefícios. Rev Cad Medicina [Internet]. 2019 [cited 2020 Jul 17];2(1):96-107. Available from: http://www.revista.unifeso.edu.br/index.php/ cadernosdemedicinaunifeso/article/view/ $1333 / 584$

Recebido: 15 de junho de 2021

Aprovado: 23 de setembro de 2021

Publicado: 10 de novembro de 2021

A Revista Baiana de Enfermagem utiliza a Licença Creative Commons - Atribuição-NãoComercial 4.0 Internacional. https://creativecommons.org/licenses/by-nc/4.0/

Este artigo é de acesso aberto distribuído sob os termos da Licença Creative Commons (CC BY-NC). Esta licença permite que outros remixem, adaptem e criem a partir do seu trabalho para fins não comerciais. Embora os novos trabalhos tenham de lhe atribuir o devido crédito e não possam ser usados para fins comerciais, os usuários não têm de licenciar esses trabalhos derivados sob os mesmos termos. 